\title{
Construction of Human Granulocyte-macrophage Colony- stimulating Factor Expression System Using Lactococcus lactis MG1363
}

\author{
Yi-Fan Hong ${ }^{1,2}$, Min Seok Ham ${ }^{3}$, Woo Jung Park ${ }^{4}$, Hangeun Kim², and Dae Kyun Chung ${ }^{1,2,5, *}$ \\ ${ }^{1}$ Graduate School of Biotechnology and Institute of Life Science and Resources, Kyung Hee University, Yongin, Gyeonggi 446-701, Korea \\ ${ }^{2}$ Skin Biotechnology Center, Kyung Hee University, Yongin, Gyeonggi 449-701, Korea \\ ${ }^{3}$ Korea Institute of Planning \& Evaluation for Technology in Food, Agriculture, Foresty \& Fisheries, Anyang, Gyeonggi 431-810, Korea \\ ${ }^{4}$ Department of Marine Food Science and Technology, Gangneung-Wonju National University, Gangneung, Gangwon 210-702, Korea \\ ${ }^{5}$ RNA Inc., \#308 College of Life Science, Kyung Hee University, Yongin, Gyeonggi 449-701, Korea
}

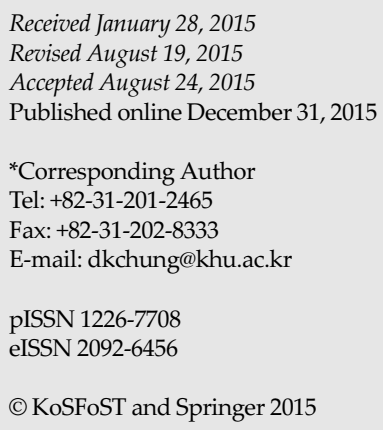

\begin{abstract}
Lactic acid bacteria (LAB) have been used in a wide variety of industrial fermentation processes. Generally, LAB have regarded as safe status, making them potentially useful organisms for the production of commercially important proteins. Here, we developed protein expression systems using Lactococcus lactis MG1363 for which the protein of interest is targeted to a defined cell location, e.g., cytoplasm, cell wall, and medium. The expression system of human granulocyte-macrophage colony-stimulating factor (hGM-CSF) was successfully constructed, and the expression of hGM-CSF in the cytoplasm of $L$. lactis was detected by Western blotting. Efficient translocation was obtained using the signal peptide from lactococcal Usp45 protein and covalently anchored to the peptidoglycan by using the cell wall anchor motif of Streptococcus pyogenes M6 protein. These results suggest that $L$. lactis containing a new expression system can deliver many other useful genes to the intestine of human and mammalian to stimulate local mucosal immunity.
\end{abstract}

Keywords: Lactic acid bacteria, Lactococcus lactis, human granulocyte-macrophage colony-stimulating factor, GM-CSF, delivery systems

\section{Introduction}

Lactic acid bacteria (LAB) including lactococci, streptococci, and lactobacilli because of their unique metabolic characteristics have been used in many fermentation processes of milk, meats, cereals, and vegetables (1). LAB and their products such as bacteriocins are considered as safe additives (Generally Recognized As Safe, GRAS) and a variety of potential beneficial effects have been published. Some of these effects include lactose digestion, cholesterol metabolism, diarrheal disorders, prevention of intestinal infections, immunomodulation, and oral vaccination (2). The intestinal regulation of $L A B$ has been also proven via numerous studies (3). $L A B$ have an ability to recruit bioactive molecules as they pass through gastrointestinal tract. By using these potential probiotic capacity of $L A B$, foreign gene encoding cytokine or antigen can be introduced into $L A B$ and induce these gene expression in the gut of human or mammalian. Transformed LAB may also be used as oral live vaccine to induce mucosal immunity (4).

Lactococcus lactis is an attractive host for the production of foreign gene because of their food grade status, efficient expression, and metabolic engineering tools. Although $L$. lactis is a nonpathogenic and non-adherent bacteria in the gastrointestinal tract, $L$. lactis when genetically modified with a recombinant plasmid can be used for the oral delivery of certain genes. Mucosal delivery of $L$. lactis expressing interleukin (IL)-2 and IL-6 accelerated immune activity (5) and treatment of murine colitis with L. lactis secreting IL10 alleviated inflammatory bowel disease (6). L. lactis producing and secreting IL-12 can be used to enhance an antigen-specific immune response and to stimulate local mucosal immunity (7). To develop a new expression systems that could be delivered to different cell fractions, L. lactis MG1363 was modified using hGM-CSF. First, hGMCSF expressing L. lactis was constructed. In this system, expressed proteins were delivered to cytoplasm. To make secreting system, signal peptide from lactococcal Usp45 protein was inserted into 5'end of hGM-CSF gene. Streptococcus pyogenes M6 protein was used to make cell wall anchor motif. The expression system, developed in this study can be used to make commercial LAB strains expressing useful cytokine. 


\section{Materials and Methods}

Bacterial strains, plasmids, and media Bacterial strains and plasmids used in this study are addressed in Table 1. E. coli XL1-blue, DH5 $\alpha$, TOP-10, and SG13009 were used as host strains for the DNA manipulation of hGM-CSF and lactococcal structure gene, and modified plasmids containing hGM-CSF was transformed into L. lactis MG1363. E. coli cells were cultured in Luria Bertani (LB) medium (1\% sodium chloride, $1 \%$ tryptone, $0.5 \%$ yeast extract) at $37^{\circ} \mathrm{C}$, shaking at 250-300 rpm. L. lactis cell culture was performed with M17 medium or Todd-Hewitt broth (Becton Dickinson, Franklin Lakes, NJ, USA) medium at $30^{\circ} \mathrm{C}$. Erythromycin $(150 \mu \mathrm{g} / \mathrm{mL})$ and ampicillin $(100 \mu \mathrm{g} /$ $\mathrm{mL}$ ) were used for $E$. coli selection, and erythromycin $(5 \mu \mathrm{g} / \mathrm{mL})$ and chloramphenicol $(10 \mu \mathrm{g} / \mathrm{mL})$ were used for the selection of L. lactis containing modified plasmid.

Construction of cytokine expression vector system hGM-CSF was amplified with specific primers (Forward: 5'-GTCGACGCACCCGCCCG CTCG-3'; Reverse: 5'-GATATCCTCCTGGACTGGCTCCCA-3') from pQE/ hGM-CSF plasmid (Cytokine Bank). PCR was performed in a total reaction volume of $50 \mu \mathrm{L}$ containing $5 \mathrm{U}$ of Taq polymerase, $1 \times$ PCR buffer, $50 \mathrm{mM} \mathrm{MgCl}, 10 \mathrm{mM}$ dNTP mixture, $10 \mu \mathrm{M}$ of each oligonucleotide primer, and $1 \mu \mathrm{L}$ of template DNA. Amplification was performed with an initial denaturation at $95^{\circ} \mathrm{C}$ for $10 \mathrm{~min}$, followed by 25 to 35 cycles at $95^{\circ} \mathrm{C}$ for $15 \mathrm{~s}, 55^{\circ} \mathrm{C}$ for $30 \mathrm{~s}$, and $72^{\circ} \mathrm{C}$ for $30 \mathrm{~s}$, and finalized at $72^{\circ} \mathrm{C}$ for $10 \mathrm{~min}$. After completion of the cycling process, PCR products were separated by $0.8 \%$ agarose gel electrophoresis. Purified PCR product was inserted into PEZ-T vector (RNA Inc., Yongin, Korea), and transformed into E. coli XL-1 blue to amplify plasmid DNA using Gene pulser unit (Bio-Rad Laboratories, Inc., Hercules, CA, USA) $(2.5 \mathrm{kV}, 25 \mu \mathrm{F}$ capacitance, $200 \Omega$ resistance). DNA sequence of inserted hGM-CSF was analyzed after plasmid preparation. $\mathrm{pEZ}-\mathrm{T} / \mathrm{hGM}$-CSF plasmids prepared from XL-1 blue were digested with Sall and EcoRV restriction enzyme, and inserted into the same restriction enzyme sites of pVE5523 (for secretion system), pVE5524 (for cell wall anchoring), and pVE5529 (for expression in cytoplasm). Constructed plasmids were transformed into $L$. lactis using Gene pulser unit ( $2.5 \mathrm{kV}, 25 \mu \mathrm{F}$ capacitance, $400 \Omega$ resistance).

L. lactis cell fractionation L. lactis containing modified plasmid was incubated with $80 \%$ ice-cold trichloroacetic acid (TAC: $16 \%$ final concentration) in ice for $20 \mathrm{~min}$ and centrifuged at $280 \times \mathrm{g}$ for $15 \mathrm{~min}$. The precipitate was washed with $1 \mathrm{~mL}$ acetone twice and dried using vacuum centrifuge. Dried pellet was resuspended with $160 \mu \mathrm{L}$ TESLMR solution (TES: 10 mM Tris-HCl pH 8.0, 1 mM EDTA, 25\% sucrose; LMR: $1 \mathrm{mg} / \mathrm{mL}$ lysozyme, $0.1 \mathrm{mg} / \mathrm{mL}$ mutanolysin, $0.1 \mathrm{mg} / \mathrm{mL}$ RNase) and incubated at $37^{\circ} \mathrm{C}$ for $30 \mathrm{~min}$ with $1 \%$ sodium dodecyl sulfate (SDS). The supernatant (containing cell wall digestion) and pellet (containing protoplast) were separated by centrifugation at $4^{\circ} \mathrm{C}$, $2,500 \times g$. The pellet containing protoplast was washed with TES solution and resuspended with $500 \mu \mathrm{L}$ distilled water. Resuspended sample was performed 5 times freeze-thawing cycle and centrifuged at $4^{\circ} \mathrm{C}, 21,000 \times \mathrm{g}$ for $45 \mathrm{~min}$ to separate cytoplasmic protein and membrane pellet. Membrane pellet was resuspended with $100 \mu \mathrm{L}$ TE solution containing 1\% SDS to get membrane protein fraction. Supernatant containing cytoplasmic protein was precipitated with TCA, and resuspended with $100 \mu \mathrm{L}$ TE solution. To obtain the cell wall digestion protein fraction, the supernatant was incubated with TCA and precipitated pellet was dissolved in $100 \mu \mathrm{L} 50 \mathrm{mM} \mathrm{NaOH}$. Medium protein fraction was prepared from $L$. lactis culture supernatant. The supernatant was filtered with $0.2 \mu \mathrm{m}$ pore-size filter, protein precipitation was performed with ice-cold $80 \%$ TCA, and the pellet was dissolved in $80 \mu \mathrm{L} 50 \mathrm{mM} \mathrm{NaOH}$.

Western blot analysis Equal amounts of protein were resolved on SDS-polyacrylamide gels (10-15\%) and then electrophoretically transferred onto nitrocellulose membrane (100 V, 2 h). Membranes were subsequently blocked with $5 \%$ skim milk in TBS-T ( $20 \mathrm{mM}$ Tris$\mathrm{HCl}, \mathrm{pH} 7.6,137 \mathrm{mM} \mathrm{NaCl}$, and 0.05\% Tween 20) and incubated with the hGM-CSF antibodies (R and D systems, Minneapolis, MN, USA). Blotting proteins were visualized by enhanced chemiluminescence (ECL) reagents (GE Healthcare, Little Chalfont, UK).

\section{Results and Discussion}

L. lactis is a food-grade bacterium that is widely used in the dairy industry. It is a potential candidate for the production of biologically useful proteins and for plasmid DNA delivery to eukaryotic cells. Several delivery systems have been developed to target heterologous proteins to a specific cell location e.g., cytoplasm, cell wall or extracellular medium, and more recently to efficiently transfer DNA to eukaryotic cells (8). Thus, protein secretion and cell wall anchoring system using $L A B$ are key factors to contribute a new functions to probiotics. GM-CSF, a monomeric glycoprotein that function as a cytokine, stimulates immune cells such as neutrophils, eosinophils, basophils, and monocytes, to produce granulocytes (9). GM-CSF also plays a role in embryonic development by functioning as an embryokine produced by reproductive tract (10). To develop a new expression system using hGM-CSF, we modified L. lactis MG1363. Amplified hGM-CSF gene was cloned into Gram-positive bacteria expression vectors. LAB expression vectors such as pVE5523, 5524, and 5529 contain P59 promoter and t1t2 and trpA terminators, which were optimized to express heterologous gene expression (11). pVE5523 contains N-terminal signal sequence of lactococcal Usp415 next to P59 promoter, which guides the expressed protein to the extracellular medium (12). pVE5524 contains C-terminal cell wall anchor domain of Streptococcus pyogenes M6 protein as well as $\mathrm{N}$-terminal signal sequence, which drives target protein to the peptidoglycan of cell wall (13). The schematic diagram of these three different vector systems was depicted in Fig. $1 \mathrm{~A}$.

Constructed plasmids were transformed into $E$. coli and erythromycin 
(A)

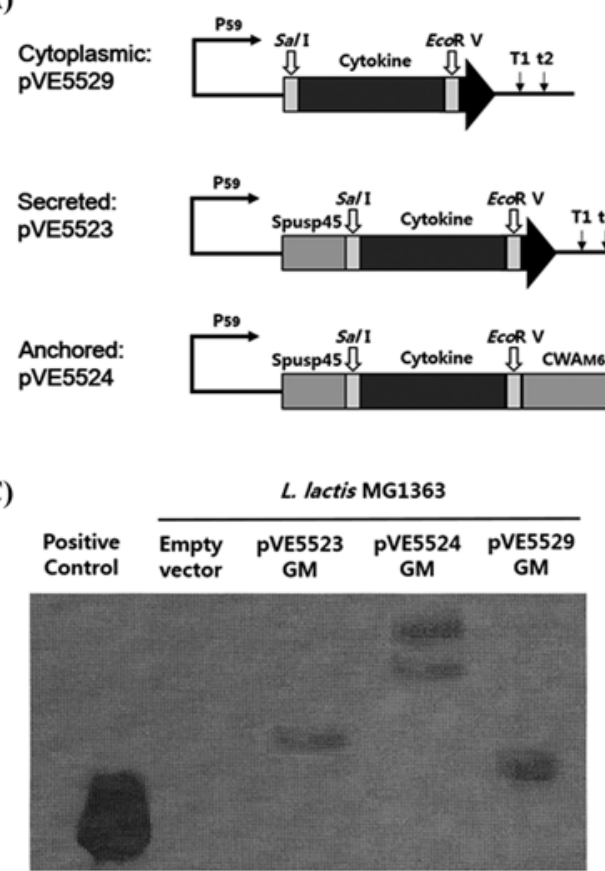

(B)

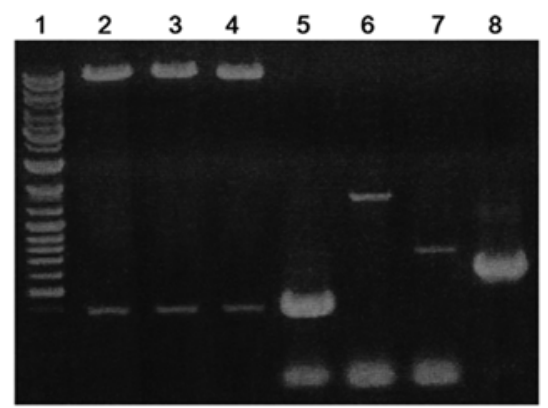

(D)

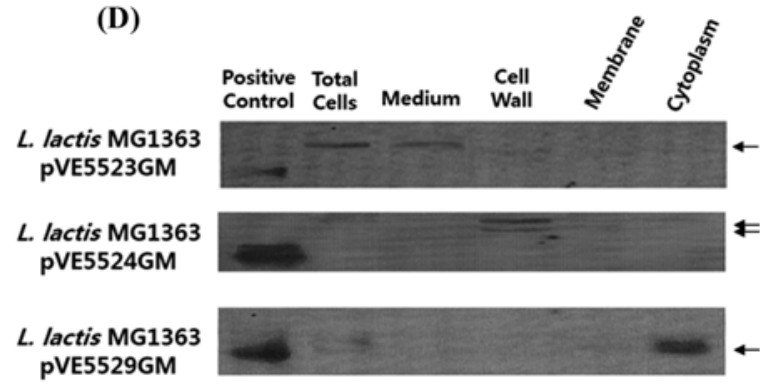

Fig. 1. Construction of expression system using L. lactis containing cytokine expression vector. (A) Schematic diagram for vector construction. (B) Confirmation of hGM-CSF cloning into Gram-positive bacteria expression vector by colony PCR and restriction enzyme digestion. 1, $1 \mathrm{~kb}$ DNA ladder; 2, pVE5524GM enzyme cut with Sall and EcoR V; 3, pVE5523GM enzyme cut with Sall and EcoR V; 4, pVE5529GM enzyme cut with Sall and ECoR V; 5, PCR product of hGM-CSF; 6, PCR product from pVE5524GM using p59 and Tt1t2 primers; 7, PCR product from pVE5523GM using p59 and Tt1t2 primers; 8, PCR product from pVE5529GM using p59 and Tt1t2 primers. (C) Western blot analysis using anti-hGM-CSF antibody of total cell lysates from L. lactis MG1363 containing pVE5523GM, pVE5524GM, or pVE5529GM. (D) Western blot analysis of cell fractions from L. lactis MG1363 containing pVE5523GM, pVE5524GM, or pVE5529GM.

resistance gene was used for the selection marker. To confirm the modified plasmid, colony PCR was performed with p59 forward primer and Tt1t2 reverse primer and transformants as a template, and Amplified plasmids in E. coli was digested with Sall and EcoRV restriction enzyme (Fig. 1B). Colony PCR result shows that PVE5523GM, 5524GM, and 5529GM produced 688, 1220, and $577 \mathrm{bp}$ PCR products, respectively. Digested product from each modified plasmids was $388 \mathrm{bp}$, whose size is consistent with hGM-CSF. DNA sequencing analysis was also continued. DNA and translated amino acid homologous search have shown that modified plasmids contain hGM-CSF gene (data not shown). The development of reliable systems for the food-grade genetic modification of $L A B$ is a very important in the development of delivery system using microorganisms. Food-grade recombinants can be used as starters in food fermentations and for the safe production of metabolites used as food adjuncts (14). The use of plasmids for food-grade introduction of new or modified genes into $L$. lactis often seems unfavorable because the presence of antibiotic resistance genes in the final constructs is not permitted and the range of alternative selection markers is limited. Several food-grade vectors have been developed by using sugar utilization (15) or the suppression of auxotrophic markers (16) for positive selection of transformants. Unfortunately, selection for these vectors requires special genotypes of the respective host strains. Thus, we need to consider an additional supplementation, e.g., integration of target gene into the bacterial genome, which may be a more reliable approach to stabilizing and maintaining the desired genetic features.

To identify the expression of cloned hGM-CSF, total cell lysates from $L$. lactis containing different modified plasmids were resolved on SDS-PAGE, and then protein expression was detected by Western blotting using anti-hGM-CSF antibody. The positive control band of hGM-CSF was appeared around 24-28 kDa. hGM-CSF protein expressed from pVE5523GM was shifted as compared to positive control band. Multiple protein bands from pVE5524GM were shown around 34 $\mathrm{kDa}$ and a single band from pVE5529GM was shown around $28 \mathrm{kDa}$ (Fig. 1C). Next, L. lactis cell fractions were subjected to Western blot analysis to identify the protein localization (Fig. 1D). As shown in the figure, major protein bands from L. lactis containing pVE5523GM were shown in total cell lysates and medium fraction, multiple bands from L. lactis containing pVE5524GM were only shown in total cell lysates and cell wall fraction, and protein bands from $L$. lactis containing pVE5529GM were appeared in total cell lysates and cytoplasm. Constructed pVE5523GM secretes hGM-CSF protein to extracellular medium in L. lactis culture. L. lactis containing pVE5524GM drives cell wall anchoring, while target protein expressed by pVE5529GM is restricted to cytoplasm. These results suggest that hGM-CSF protein expression was successfully targeted on their right position according to the constructed plasmids, and indicating that the 
Table 1. Bacteria strains and plasmids used in this study

\begin{tabular}{|c|c|c|}
\hline Strain or plasmid & Relevant characteristic & Reference \\
\hline \multicolumn{3}{|l|}{ Strain } \\
\hline E. coli XL1-Blue & Plasmid-free strain & \\
\hline $\mathrm{DH} 5 \alpha$ & Plasmid-free strain & \\
\hline TOP-10 & Plasmid-free strain & \\
\hline SG13009 & Plasmid-free strain & Qiagen \\
\hline L. lactis MG1363 & Plasmid-free strain & \\
\hline \multicolumn{3}{|l|}{ Plasmid } \\
\hline pQE/hGM-CSF & Ap ${ }^{r} ;$ ColE1; 3.9 kb :: hGM-CSF & Cytokine Bank \\
\hline $\mathrm{pUC18/hG-CSF}$ & Ap ${ }^{r} ;$ ColE1; 3.5 kb :: hG-CSF & Cytokine Bank \\
\hline $\mathrm{pUC18} / \mathrm{hIFN}-\gamma$ & Apr; ColE1; $3.1 \mathrm{~kb}::$ hIFN- $\gamma$ & Cytokine Bank \\
\hline pEZ-T & $A p^{r} ;$ ColE1; $3.0 \mathrm{~kb}$ & RNA Inc. \\
\hline pVE5523 & $A p^{r} ; E^{r} ;$ ColE1; pAM $\beta 1 ; p B S:: p I L 252:: t_{\text {trp }} A:: P_{59}:: S P U s p 45::$ nucA::t1t2 & $(11)$ \\
\hline pVE5524 & $A p^{r} ; E^{r} ;$ ColE1; pAM $\beta 1 ;$ pBS::plL252::t trp $A:: P_{59}:: S P U s p 45::$ nucA::CWA ${ }_{M 6}:: t 1 t 2$ & (11) \\
\hline pVE5529 & $A p^{r} ; E^{r} ;$ ColE1; pAM $\beta 1 ; p B S:: p I L 252:: t_{\text {trp }} A:: P_{59}::$ nucA::t1t2 & (11) \\
\hline pVE5523GM, G, I & $A p^{r} ; E^{r} ;$ ColE1; pAM $\beta 1 ; p B S:: p I L 252:: t_{\text {trp }} A:: P_{59}::$ cytokine::nucA::t1t2 & This work \\
\hline pVE5524GM, G, I & $A p^{r} ; E^{r} ;$ ColE1; pAM $\beta 1 ;$ pBS::plL252::t trp $A:: P_{59}:: c y t o k i n e:: n u c A:: C W A_{M 6}:: t 1 t 2$ & This work \\
\hline pVE5529GM, G, I & $A p^{r} ; E^{r} ;$ ColE1; pAM $\beta 1 ; p B S:: p I L 252:: t_{\text {trp }} A:: P_{59}:: c y$ tokine::t1t2 & This work \\
\hline
\end{tabular}

ColE1, replicon from E. coli plasmid; pAM $\beta 1$, replicon from Gram positive bacteria; Ap ${ }^{r}$, ampicillin resistance; Em ${ }^{r}$, erythromycin resistance

cytokine expression system can be used for the developing of commercially useful LAB strains. Administration of LAB strains carrying the cytokine-cloned plasmid will be effective at diminishing inflammation or stimulating immune cells against infected pathogens.

All of the L. lactis strains that have been developed to be used as vaccine DNA delivery vectors are promising for mucosal immunization. Recently, however, several studies have shown that recombinant $L$. lactis can be used as a therapeutic vector such as the treatment of few Crohn's disease patients $(17,18)$. In this study, we also constructed the expression vector system using human Granulocyte-colony stimulating factor (hG-CSF) and human interferon-gamma (hIFN- $\gamma$ ) genes (data not shown). Unfortunately, in this study, we did not confirm the activity of $L$. lactis expressing human cytokines against the immune modulation. Thus, additional experiments should be performed to test activity and safety of invented delivery system. For example, the physiological effect of L. lactis expressing hGM-CSF or hG-CSF can be examined with the proliferation of AML-193 cell line and the antiviral activity of IFN- $\gamma$ expressed $L$. lactis can be tested with $A 549$ cells containing encephalomyocarditis virus (EMCV). Oral administration test using modified $L$. lactis should be carried out to confirm the safety. The delivery of pathogen-derived vaccine through oral or nasal may provoke local immune responses at the entry. However, the use of $L$. lactis to deliver hGM-CSF to a mucosal surface may reduce toxic side effects and have clear advantages of low-cost and simple method of administration. Taken together, our study suggest that $L$. lactis containing a new expression system can deliver many other useful genes to the intestine of human and mammalian.

Acknowledgment This work was supported by a grant from Kyung Hee University in 2012 (KHU-20120816).
Disclosure The authors declare no conflict of interest.

\section{References}

1. Caplice E, Fitzgerald GF. Food fermentations: Role of microorganisms in food production and preservation. Int. J. Food Microbiol. 50: 131-149 (1999)

2. Marteau P, Rambaud JC. Potential of using lactic acid bacteria for therapy and immunomodulation in man. FEMS Microbiol. Rev. 12: 207-220 (1993)

3. Salminen S, Bouley C, Boutron-Ruault MC, Cummings JH, Franck A, Gibson GR, Isolauri E, Moreau MC, Roberfroid M, Rowland I. Functional food science and gastrointestinal physiology and function. Brit. J. Nutr. 80: S147-S171 (1998)

4. Holmgren J, Czerkinsky C, Lycke N, Svennerholm AM. Mucosal immunity: implications for vaccine development. Immunobiology 184: 157-179 (1992)

5. Steidler L, Robinson K, Chamberlain L, Schofield KM, Remaut E, Le Page RW, Wells JM. Mucosal delivery of murine interleukin-2 (IL-2) and IL-6 by recombinant strains of Lactococcus lactis coexpressing antigen and cytokine. Infect. Immun. 66: 3183-3189 (1998)

6. Steidler L, Hans W, Schotte L, Neirynck S, Obermeier F, Falk W, Fiers W, Remaut E. Treatment of murine colitis by Lactococcus lactis secreting interleukin-10. Science 289: 1352-1355 (2000)

7. Bermúdez-Humarán LG, Langella $P$, Cortes-Perez NG, Gruss $A$, Tamez-Guerra RS, Oliveira SC, Saucedo-Cardenas O, Montes de Oca-Luna R, Le Loir Y. Intranasal immunization with recombinant Lactococcus lactis secreting murine interleukin-12 enhances antigen-specific Th1 cytokine production. Infect. Immun. 71: 1887-1896 (2003)

8. Pontes DS, de Azevedo MS, Chatel JM, Langella P, Azevedo V, Miyoshi A. Lactococcus lactis as a live vector: Heterologous protein production and DNA delivery systems. Protein Expres. Purif. 79: 165-175 (2011).

9. Francisco-Cruz A, Aguilar-Santelises M, Ramos-Espinosa O, Mata-Espinosa D, Marquina-Castillo B, Barrios-Payan J, Hernandez-Pando R. Granulocytemacrophage colony-stimulating factor: Not just another haematopoietic growth factor. Med. Oncol. 31: 774 (2014)

10. Hansen PJ, Dobbs KB, Denicol AC. Programming of the preimplantation embryo by the embryokine colony stimulating factor 2 . Anim. Reprod. Sci. 149: 59-66 (2014)

11. Dieye Y, Hoekman AJ, Clier F, Juillard V, Boot HJ, Piard JC. Ability of Lactococcus lactis to export viral capsid antigens: A crucial step for development of live vaccines. Appl. Environ. Microb. 69: 7281-7288 (2003)

12. Borrero J, Jiménez JJ, Gútiez L, Herranz C, Cintas LM, Hernández PE. Use of the usp45 lactococcal secretion signal sequence to drive the secretion and functional expression of enterococcal bacteriocins in Lactococcus lactis. Appl. 
Microbiol. Biot 89: 131-143 (2011)

13. Piard JC, Hautefort I, Fischetti VA, Ehrlich SD, Fons M, Gruss A. Cell wal anchoring of the Streptococcus pyogenes M6 protein in various lactic acid bacteria. J. Bacteriol. 179: 3068-3072 (1997)

14. Henrich B, Klein JR, Weber B, Delorme C, Renault P, Wegmann U. Food-grade delivery system for controlled gene expression in Lactococcus lactis. Appl. Environ. Microb. 68: 5429-5436 (2002)

15. Platteeuw $C$, van Alen-Boerrigter I, van Schalkwijk S, de Vos WM. Food-grade cloning and expression system for Lactococcus lactis. Appl. Environ. Microb. 62: 1008-1013 (1996)
16. Dickely, F, Nilsson D, Hansen EB, Johansen E. Isolation of Lactococcus lactis nonsense suppressors and construction of a food-grade cloning vector. Mol. Microbiol. 15: 839-847 (1995)

17. Braat $H$, Rottiers $P$, Hommes $D W$, Huyghebaert $N$, Remaut $E$, Remon JP, van Deventer SJ, Neirynck S, Peppelenbosch MP, Steidler L. A phase I trial with transgenic bacteria expressing interleukin-10 in Crohn's disease. Clin. Gastroenterol. H. 4: 754-759 (2006)

18. Rottiers P, De Smedt T, Steidler L. Modulation of gut-associated lymphoid tissue functions with genetically modified Lactococcus lactis. Int. Rev. Immunol. 28: 465-486 (2009) 